\title{
UNA NUEVA ESPECIE DE Microtus (Allophaiomys) (ARVICOLIDAE, RODENTIA, MAMMALIA) EN EL PLEISTOCENO INFERIOR DE LA SIERRA DE ATAPUERCA (BURGOS, ESPAÑA)
}

\author{
César LAPLANA y Gloria CUENCA-BESCÓS
}

\author{
Dpto. de Ciencias de la Tierra (Paleontología), Facultad de Ciencias, Universidad de Zaragoza (UA \\ CSIC). Ciudad Universitaria. 50.009 Zaragoza.
}

\begin{abstract}
Laplana, C. y Cuenca-Bescós, G. 2000. Una nueva especie de Microtus (Allophaiomys) (Arvicolidae, Rodentia, Mammalia) en el Pleistoceno Inferior de la Sierra de Atapuerca (Burgos, España). [A new Lower Pleistocene Microtus (Allophaiomys) species (Arvicolidae, Rodentia, Mammalia) from the Sierra de Atapuerca (Burgos, Spain).] Revista Española de Paleontología, 15(1), 77-87. ISSN 0213-6937.
\end{abstract}

\begin{abstract}
A new species of Microtus (Allophaiomys), M. (A.) lavocati nov. sp. is described from the Sima del Elefante (Sierra de Atapuerca, Burgos, Spain) karst infilling. The biometrical analysis carried out on the first lower molars using 19 variables allows its separation from other southern and western-European species of Microtus (Allophaiomys), as M. (A.) pliocaenicus, M. (A.) hintoni nutiensis, M. (A.) burgondiae, M. (A.) valerotensis and M. (A.) cf. ruffoi. $M$. (A.) lavocati nov. sp. is characterized by a great development of the anterior part of the first lower molar, by the wide communication of triangles T4 and T5 and by having and inclined T4 triangle. This is one of the so far youngest known species of Microtus (Allophaiomys), as suggested by the Sima del Elefante rodent association and by its own morphological characteristics.
\end{abstract}

Keywords: Microtus (Allophaiomys), Lower Pleistocene, Sierra de Atapuerca, Spain.

\section{RESUMEN}

Se describe una nueva especie de Microtus (Allophaiomys), M. (A.) lavocati nov. sp., a partir de material del relleno cárstico Sima del Elefante de la Sierra de Atapuerca (Burgos). El análisis biométrico realizado utilizando 19 variables medidas sobre los primeros molares inferiores permite su separación de las especies $M$. (A.) pliocaenicus, $M$. (A.) hintoni nutiensis, $M$. (A.) burgondiae, $M$. (A.) valerotensis y $M$. (A.) cf. ruffoi, presentes en el sur y oeste de Europa. M. (A.) lavocati nov. sp. se caracteriza por un gran desarrollo de la parte anterior del primer molar inferior junto a una amplia comunicación de los triángulos T4 y T5 y un triángulo T4 inclinado. La asociación de roedores que acompaña a esta especie en la Sima del Elefante junto a sus propias características morfológicas sugieren que se trata de una de las especies más modernas de Microtus (Allophaiomys) conocidas hasta ahora.

Palabras clave: Microtus (Allophaiomys), Pleistoceno Inferior, Sierra de Atapuerca, España.

\section{INTRODUCCIÓN}

Las calizas cretácicas carstificadas de la Sierra de Atapuerca (Burgos) albergan numerosas cavidades, la mayor parte de ellas rellenadas de sedimentos a lo largo del Pleistoceno. Algunos de estos rellenos, seccionados por la trinchera de un antiguo ferrocarril minero, afloran hoy día en superficie, como los denominados Gran Dolina y complejo Galería-Tres Simas. Los sedimentos que forman estos rellenos son especialmente ricos en registro paleontológico de vertebrados y constituyen yacimientos de referencia para el Cuaternario ibérico.
Uno de los rellenos atravesado por la trinchera del ferrocarril es el llamado Sima del Elefante. Este relleno se sitúa unos $100 \mathrm{~m}$ al sur de los antes citados, y sus sedimentos afloran a ambos lados de la trinchera. Desde el punto de vista estratigráfico, el relleno de la Sima del Elefante, con un espesor visible en sección de unos $11 \mathrm{~m}$, se puede dividir en tres grandes unidades: una unidad roja inferior, de aproximadamente $3 \mathrm{~m}$ de espesor, compuesta por arcillas con o sin bloques de caliza, con algunos niveles de murcielaguina, muy rica en restos fósiles; una unidad blanca intermedia, de unos $4 \mathrm{~m}$ de espesor, constituida por una brecha de bloques de caliza con 


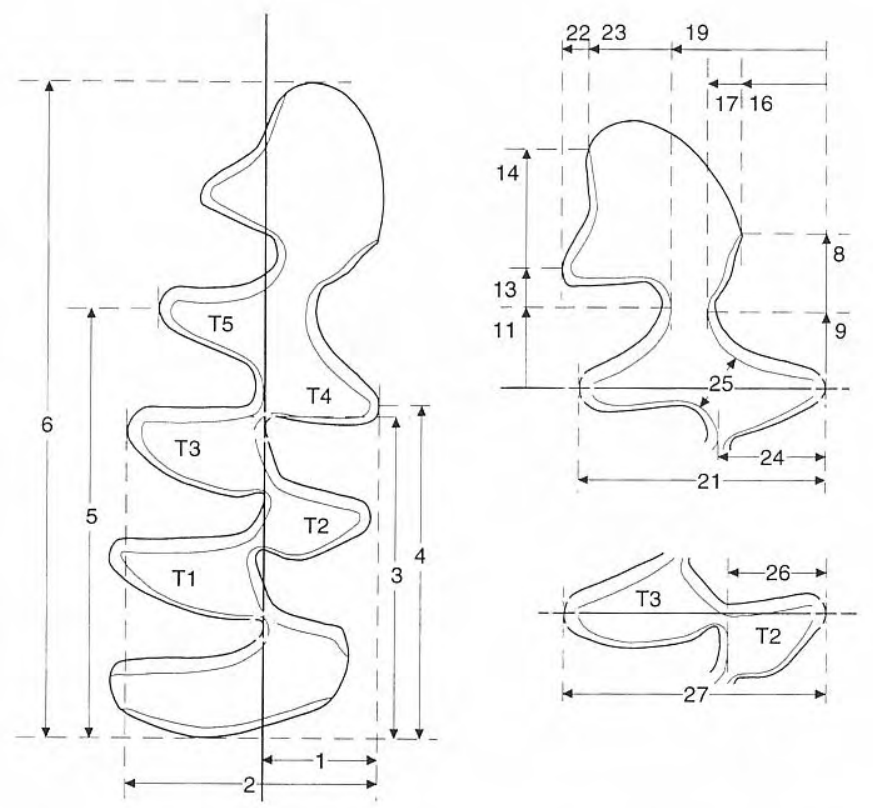

Figura 1. Esquema de las medidas tomadas sobre los primeros molares inferiores (m1) de Allophaiomys.

matriz limosa, con algunos niveles de travertinos; y una unidad roja superior, de aproximadamente $4 \mathrm{~m}$ de potencia, formada por coladas de bloques con matriz arcillosa ocasionalmente cementadas. La única datación realizada en este relleno se ha llevado a cabo sobre uno de los niveles travertínicos de la unidad blanca intermedia, por el método de las series del uranio, con un resultado de $213 \pm 20 \mathrm{Ka}$ (Bischoff, $1991 \mathrm{com}$. pers. en Carbonell et al., 1995).

Desde 1992 se vienen realizando muestreos en este yacimiento, con el objetivo de conocer su contenido en microvertebrados y poder utilizarlos como elemento de datación. En 1995 se inició un muestreo sistemático de la parte inferior del relleno (unidad roja inferior), con la realización de una excavación reducida de $1 \mathrm{~m}^{2}$ de extensión, ampliada a $2 \mathrm{~m}^{2}$ en 1996. En 1996 se realizó además un sondeo con excavadora, de $5 \mathrm{~m}$ de profundidad, de la parte del relleno situada por debajo de la superficie del piso de la trinchera. Este sondeo también fue muestreado en busca de microfauna. Los trabajos se han continuado a lo largo de los últimos años y en la actualidad la excavación se encuentra muy próxima al nivel del piso de la trinchera.

Los sedimentos de la unidad roja inferior se han revelado muy ricos en microvertebrados, con una excelente conservación. Las muestras tomadas en distintos niveles de esta unidad son homogéneas desde el punto de vista de la composición de la microfauna, con variaciones que atañen únicamente a las especies menos representadas. Los roedores identificados hasta el momento son: Microtus (Allophaiomys) chalinei Alcalde, Agustí y Villata, 1981, Microtus (Iberomys) aff. huescarensis (Ruiz Bustos, 1988), Ungaromys nanus Kormos, 1933, Pliomys episcopalis Méhely, 1914, Mimomys sp., Castillomys rivas Martín Suárez y Mein,

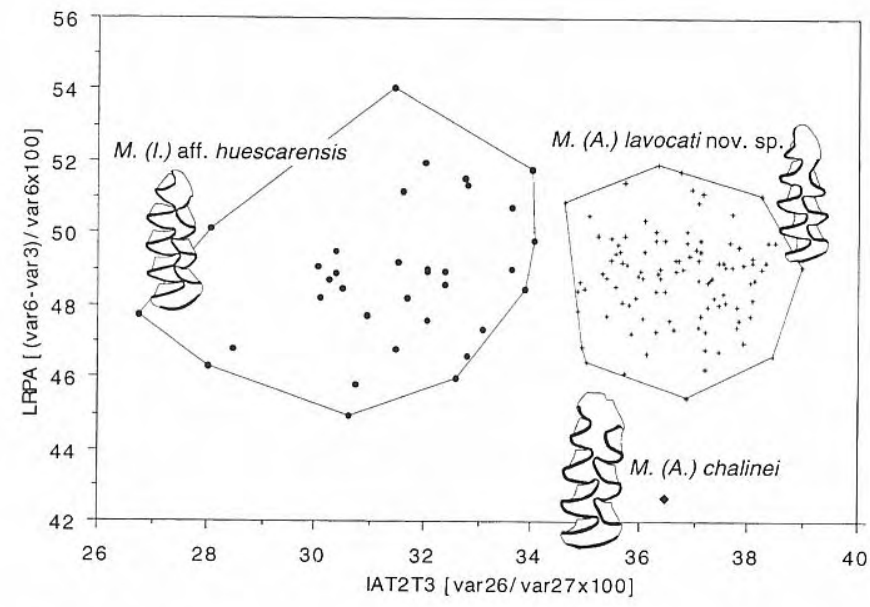

Figura 2. Distribución de las especies de Microtus de la unidad roja inferior de la Sima del Elefante respecto a las variables LRPA (eje vertical) y IAT2T3 (eje horizontal). El índice de asimetría IAT2T3 separa $M$. (A.) aff. huescarensis de las otras dos especies, ya que ésta se caracteriza por m1 con una elevada asimetría transversal. El índice LRPA, que mide el desarrollo relativo de la región anterior del molar, separa $M$. (A.) chalinei de las otras dos especies, ya que esta especie tiene la parte anterior del $\mathrm{ml}$ poco desarrollada. La dispersión de la nube de $M$. (I.) aff. huescarensis es mayor que la de $M$. (A.) lavocati nov. sp. a pesar de contar con un número inferior de individuos debido a que los ejemplares de la primera especie provienen de varios niveles, mientras que los de la segunda son todos de un solo nivel.

1991, Apodemus sp., Eliomys quercinus (L., 1766), Myoxus sp., Castor fiber (L., 1758). Junto a estas especies se ha reconocido otro arvicólido de características singulares, que es considerado aquí como una nueva especie: Microtus (Allophaiomys) lavocati nov. sp.

\section{MATERIAL Y MEDIDAS}

Se ha comparado la morfología de Allophaiomys de la Sima del Elefante con la de otras especies de Allophaiomys morfológica y geográficamente próximas, como M. (A.) pliocaenicus (Kormos, 1933) de Betfia II (Rumanía), M. (A.) hintoni nutiensis (Chaline, 1972) $M$. (A.) burgondiae (Chaline, 1972) y M. (A.) valerotensis Laplana et al., en prensa, de Les Valerots (Francia), y $M$. (A.) cf. ruffoi (Pasa, 1947) de Cava Pirro (Italia). La población de $M$. (A.) pliocaenicus de Betfia II constituye la población tipo de esta especie y con posterioridad a su definición ha sido descrita por Van der Meulen (1973) y más recientemente por Hír (1998). Las especies de Les Valerots fueron definidas y descritas por Chaline (1972) y han sido objeto de reciente revisión por Laplana et al. (en prensa). Las poblaciones de $M$. (A.) cf. ruffoi de Cava Pirro han sido descritas por Masini y Santini (1991). De 


\begin{tabular}{l|rrrr}
\hline & eje 1 & eje 2 & eje 3 & eje 4 \\
\hline var3 & 0,917 & $-0,118$ & $-0,078$ & 0,227 \\
var4 & 0,817 & $-0,092$ & $-0,180$ & 0,351 \\
var5 & 0,914 & $-0,082$ & $-0,138$ & 0,196 \\
var6 & 0,925 & 0,192 & $-0,051$ & 0,202 \\
var8 & 0,580 & $-0,132$ & $-0,493$ & 0,153 \\
var9 & 0,541 & 0,578 & 0,221 & $-0,165$ \\
$\operatorname{var11}$ & 0,633 & 0,613 & $-0,055$ & $-0,013$ \\
$\operatorname{var13}$ & 0,618 & $-0,220$ & $-0,349$ & 0,027 \\
$\operatorname{var14}$ & 0,360 & 0,701 & $-0,212$ & 0,321 \\
$\operatorname{var16}$ & 0,572 & $-0,398$ & $-0,483$ & $-0,217$ \\
$\operatorname{var17}$ & 0,594 & 0,440 & $-0,042$ & $-0,440$ \\
$\operatorname{var19}$ & 0,766 & $-0,455$ & 0,138 & $-0,216$ \\
$\operatorname{var21}$ & 0,939 & -0.033 & 0,047 & $-0,046$ \\
$\operatorname{var22}$ & 0,829 & 0,257 & 0,111 & $-0,237$ \\
$\operatorname{var23}$ & 0,714 & $-0,181$ & 0,345 & $-0,417$ \\
$\operatorname{var24}$ & 0,833 & $-0,126$ & 0,335 & 0,027 \\
$\operatorname{var25}$ & 0,130 & $-0,113$ & 0,683 & 0,553 \\
$\operatorname{var26}$ & 0,804 & $-0,196$ & 0,285 & $-0,013$ \\
$\operatorname{var27}$ & 0,923 & $-0,132$ & 0,110 & 0,021 \\
\hline
\end{tabular}

cada una de las especies consideradas se han medido 50 ejemplares.

Las medidas de las poblaciones de Les Valerots y de la Sima del Elefante han sido tomadas utilizando un equipo Nikon Measurescope en el laboratorio de paleontología de la Universidad de Borgoña (Francia). En el caso de la población de Betfia II se han utilizado dibujos inéditos realizados por el prof. A. Van der Meulen existentes en el laboratorio de paleontología de la Universidad de Borgoña, a los que hemos tenido acceso gracias a P. Brunet-Lecomte, S. Montuire y al prof. J. Chaline. Para la población de Cava Pirro se han utilizado los dibujos de la publicación de este material realizados por Masini y Santini (1991).

\section{METODOLOGÍA}

En el análisis morfométrico de los primeros molares inferiores $(\mathrm{m} 1)$ se ha utilizado la metodología propuesta por Brunet-Lecomte (1988) utilizada para el estudio de los representantes actuales y fósiles de Terricola, con algunas modificaciones. Dada la simplicidad del modelo dentario de Allophaiomys, las medidas 7, 10, 12, 15, 18, 20 han sido eliminadas. Por contra, han sido añadidas nuevas medidas $(24,25,26$ y 27). Las medidas 26 y 27 son similares a las 1 y 2 del esquema original, y las sustituyen. En total, se han considerado 19 medidas sobre cada m1 (Fig. 1).

A partir de estas medidas se han calculado algunos índices en relación con determinadas características morfológicas de los molares. El índice LRPA [(var6var3)/var6x100], análogo al índice A/L propuesto por Van

\begin{tabular}{l|cc}
\hline & varianza & $\%$ \\
\hline eje 1 & 10,29 & 54,2 \\
eje 2 & 2,08 & 10,9 \\
eje 3 & 1,58 & 8,3 \\
eje 4 & 1,24 & 6,5 \\
\hline
\end{tabular}

Tabla 1. Resultados del análisis de componentes principales. Vectores y valores propios.

der Meulen (1973), expresa el desarrollo relativo de la parte anterior del molar; el índice RP [(var4var3)/var6x100] expresa la inclinación del triángulo T4; el índice ICAC2RP [(var19-var17)/var21x100] expresa la comunicación entre el rombo pitimiano y el lóbulo anterior, y es análogo al índice B propuesto por Van der Meulen (op. cit.); el índice IAT2T3 [var26/var27x 100] expresa la asimetría transversal del molar medida sobre los triángulos T2 y T3; y el índice ICT4T5 [var25/var21x 100] expresa la confluencia entre los triángulos T4 y T5, y es análogo al índice $\mathrm{C}$ propuesto por Van der Meulen (op. cit.). Los índices LRPA y RP han sido utilizados por Brunet-Lecomte (1988) y los ICAC2RP y IAT2T3 por Laplana et al. (en prensa).

La comparación de la morfología de los primeros molares inferiores de las distintas especies estudiadas se ha realizado mediante análisis de componentes principales (ACP) realizados sobre las medidas originales, y mediante la comparación de las medias de los índices calculados y de determinadas medidas en particular, utilizando para ello análisis de la varianza (ANOVA) acompañados del test de Scheffe.

\section{SISTEMÁTICA}

En la unidad roja inferior del relleno cárstico Sima del Elefante se encuentran tres formas distintas de Microtus; la primera de ellas, representada por $\mathrm{m} 1$ con molares relativamente asimétricos, triángulos con los extremos apuntados y complejos anteriores con morfologías de tipo "hintoni", asignada a M. (Iberomys) aff. huescarensis; la segunda, de gran talla, con un complejo anterior de desarrollo reducido con un lóbulo anterior muy simple, y con el esmalte indiferenciado, características de $M$. (A.) chalinei; y la tercera, con una morfología típica de Allophaiomys, es decir, con $\mathrm{m} 1$ con los triángulos $\mathrm{T} 4 \mathrm{y}$ T5 confluyentes entre sí y con el lóbulo anterior, éste último con una estructura muy simple con T6 y T7 poco desarrollados. La Fig. 2 permite apreciar que la separación entre estas formas es sencilla, utilizando como criterios discriminadores la asimetría de los $\mathrm{m} 1$ medida sobre los triángulos T2 y T3 y el desarrollo relativo de la parte anterior del molar.

Para establecer la entidad taxonómica de esta forma, se ha realizado la comparación con las especies de Allophaiomys próximas en morfología y cronología antes indicadas. El ACP, realizado sobre 50 ejemplares de cada una de las especies indicadas, retiene 4 ejes, aunque sólo 

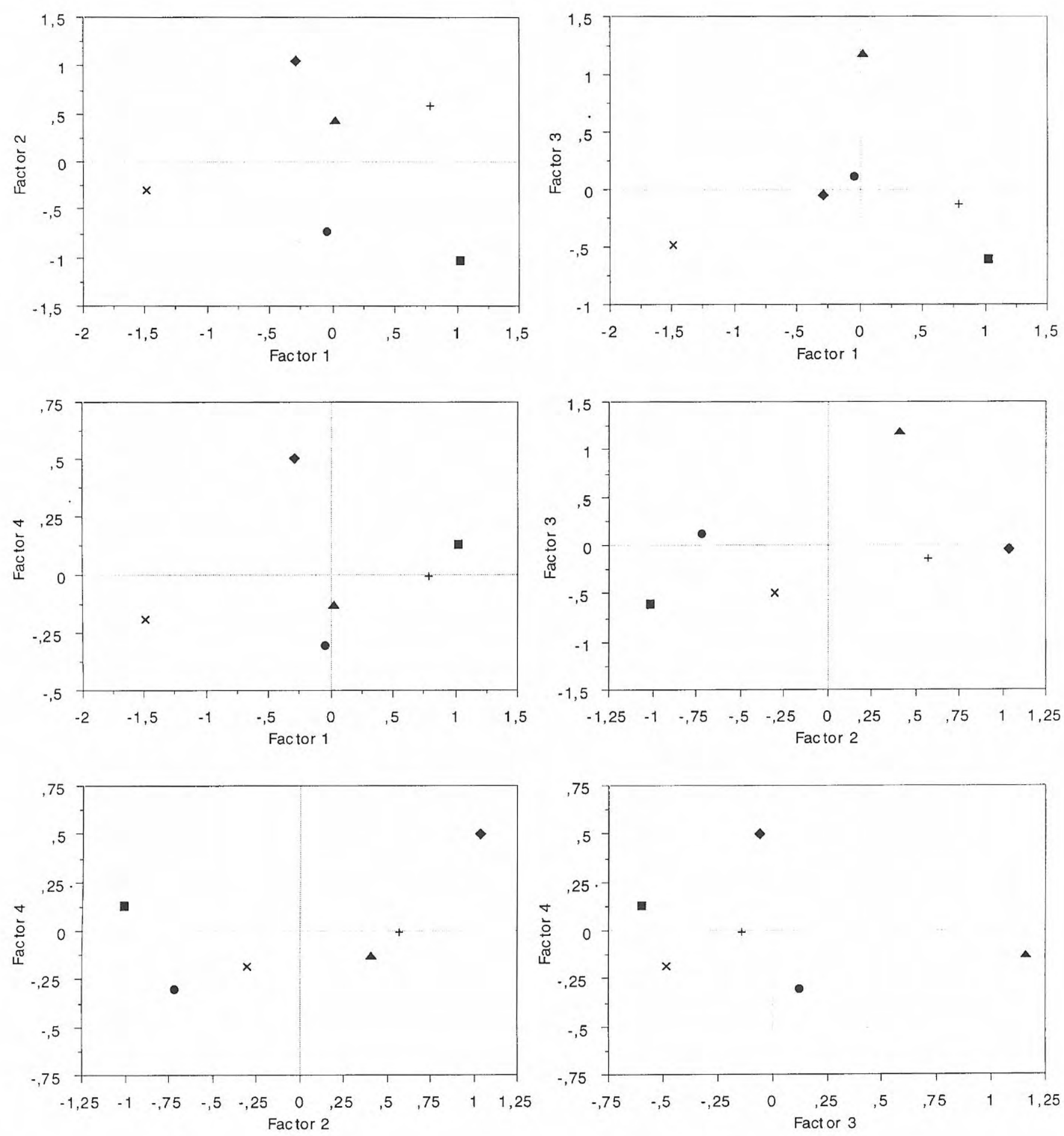

- Microtus (Allophaiomys) pliocaenicus

- Microtus (Allophaiomys) hintoni nutiensis

+ Microtus (Allophaiomys) burgondiae

$\times$ Microtus (Allophaiomys) valerotensis

- Microtus (Allophaiomys) cf. ruffoi

- Microtus (Allophaiomys) lavocati nov. sp.

Figura 3. Centroides de las distribuciones de las poblaciones de Allophaiomys consideradas sobre los planos definidos por los factores retenidos. Se aprecia que el tercer factor opone $M$. (A.) lavocati nov. sp. a las demás especies.

el primero expresa un porcentaje de varianza superior al $15 \%(54,2 \%)$. Este primer eje puede considerarse una medida del tamaño de los molares, ya que todas las variables utilizadas presentan una alta correlación positiva con el mismo (Tabla 1). Los siguientes ejes están relacionados con la morfología de $\operatorname{los} \mathrm{m} 1$, ya que presentan altas correlaciones (positivas o negativas) sólo con determinadas variables. 


\begin{tabular}{l|c|cccccc}
\hline & $\mathbf{n}$ & $\mathbf{v 6}(\mathbf{m m})$ & LRPA (s.u.) & $\mathbf{R P}$ (s.u.) & $\begin{array}{c}\text { ICAC2RP } \\
\text { (s.u.) }\end{array}$ & $\begin{array}{c}\text { IAT2T3 } \\
\text { (s.u.) }\end{array}$ & $\begin{array}{c}\text { ICT4T5 } \\
\text { (s.u.) }\end{array}$ \\
\hline M. (A.) pliocaenicus & 50 & $2,58 \pm 0,17$ & $44,33 \pm 2,14$ & $2,24 \pm 1,43$ & $21,04 \pm 6,75$ & $37,33 \pm 1,87$ & $20,03 \pm 3,80$ \\
M. (A.) nutiensis & 50 & $2,65 \pm 0,11$ & $47,26 \pm 1,68$ & $3,51 \pm 1,30$ & $6,88 \pm 5,98$ & $34,47 \pm 2,17$ & $20,76 \pm 4,60$ \\
M. (A.) burgondiae & 50 & $2,81 \pm 0,13$ & $45,80 \pm 1,57$ & $2,19 \pm 1,50$ & $14,78 \pm 5,73$ & $36,08 \pm 1,57$ & $18,34 \pm 4,41$ \\
M. (A.) valerotensis & 50 & $2,34 \pm 0,10$ & $48,45 \pm 1,38$ & $4,69 \pm 1,22$ & $18,31 \pm 4,50$ & $35,52 \pm 1,71$ & $19,77 \pm 3,82$ \\
M. (A.) cf. ruffoi & 50 & $2,78 \pm 0,16$ & $43,35^{ \pm} 1,88$ & $1,01 \pm 1,35$ & $26,67 \pm 5,58$ & $35,20 \pm 1,79$ & $18,47 \pm 4,41$ \\
M. (A.) lavocati & 50 & $2,65 \pm 0,11$ & $48,74 \pm 1,38$ & $0,97 \pm 1,45$ & $21,08 \pm 4,23$ & $36,81 \pm 1,25$ & $24,34 \pm 3,24$ \\
\hline ANOVA & & $\mathrm{p}<0,0001$ & $\mathrm{p}<0,0001$ & $\mathrm{p}<0,0001$ & $\mathrm{p}<0,0001$ & $\mathrm{p}<0,0001$ & $\mathrm{p}<0,0001$ \\
\hline
\end{tabular}

Tabla 2. Medias, desviación estándar y ANOVA de la longitud (v6) y de los índices propuestos en relación con la morfología de $\operatorname{los} \mathrm{m} 1$.

\begin{tabular}{|c|c|c|c|c|c|c|c|c|c|c|c|c|}
\hline & var6 & & LRPA & & $\mathbf{R P}$ & & ICAC2RI & & LAT2T3 & & \multicolumn{2}{|l|}{ ICT4T5 } \\
\hline M. (A.) lavocat.M. (A.) plioc. & 0,3517 & & $<0,0001$ & & 0,0008 & $\mathrm{~S}$ & $>0,9999$ & & 0,8110 & & $<0,0001$ & \\
\hline M. (A.) lavocat.M. (A.) nutiensis & $>0,9999$ & & 0,0022 & $\mathrm{~s}$ & $<0,0001$ & $\mathrm{~s}$ & $<0,0001$ & $\mathrm{~s}$ & $<0,0001$ & $\mathrm{~S}$ & 0,0015 & S \\
\hline M. (A.) lavocat.M. (A.) burgond. & $<0,0001$ & $\mathrm{~S}$ & $<0,0001$ & $\mathrm{~s}$ & 0,0016 & $\mathrm{~s}$ & $<0,0001$ & $\mathrm{~s}$ & 0,4650 & & $<0,0001$ & $\mathrm{~S}$ \\
\hline M. (A.) lavocat.-M. (A.) valerot. & $<0,0001$ & $\mathrm{~S}$ & 0,9799 & & $<0,0001$ & $S$ & 0,3658 & & 0,0009 & $\mathrm{~s}$ & $<0,0001$ & $\mathrm{~S}$ \\
\hline M. (A.) lavocati-M. (A.) cf. ruffoi & 0,0003 & $\mathrm{~S}$ & $<0,0001$ & $\mathrm{~S}$ & $>0,9999$ & & 0,0002 & $\mathrm{~s}$ & 0,0006 & $\mathrm{~s}$ & $<0,0001$ & $\mathrm{~S}$ \\
\hline M. (A.) plioc.-M. (A.) nutiensis & 0,3061 & & $<0,0001$ & $\mathrm{~S}$ & 0,0005 & $\mathrm{~s}$ & $<0,0001$ & s & $<0,0001$ & $\mathrm{~S}$ & 0,9744 & \\
\hline M. (A.) plioc.-M. (A.) burgondiae & $<0,0001$ & $\mathrm{~S}$ & 0,0026 & $\mathrm{~s}$ & $>0,9999$ & & $<0,0001$ & $\mathrm{~s}$ & 0,0221 & $\mathrm{~S}$ & 0,4792 & \\
\hline M. (A.) plioc.-M. (A.) valerot. & $<0,0001$ & $\mathrm{~S}$ & $<0,0001$ & $\mathrm{~S}$ & $<0,0001$ & $\mathrm{~S}$ & 0,3854 & & $<0,0001$ & $\mathrm{~S}$ & $>0,9999$ & \\
\hline M. (A.) plioc.-M. (A.) cf. ruffoi & $<0,0001$ & $\mathrm{~S}$ & 0,1404 & & 0,0014 & S & 0,0002 & $\mathrm{~s}$ & $<0,0001$ & $\mathrm{~S}$ & 0,5752 & \\
\hline M. (A.) nutien.-M. (A.) burgond. & $<0,0001$ & $\mathrm{~S}$ & 0,0028 & $\mathrm{~S}$ & 0,0002 & $\mathrm{~s}$ & $<0,0001$ & $\mathrm{~s}$ & 0,0003 & $S$ & 0,1033 & \\
\hline M. (A.) nutien.M. (A.) valerot. & $<0,0001$ & $\mathrm{~S}$ & 0,0329 & $\mathrm{~S}$ & 0,0007 & $\mathrm{~S}$ & $<0,0001$ & $\mathrm{~S}$ & 0,1920 & & 0,9901 & \\
\hline M. (A.) nutien.-M. (A.) cf. ruffoi & 0,0005 & $\mathrm{~S}$ & $<0,0001$ & $\mathrm{~S}$ & $<0,0001$ & $\mathrm{~S}$ & $<0,0001$ & S & 0,2308 & & 0,1470 & \\
\hline M. (A.) burgond.-M. (A.) valerot. & $<0,0001$ & $\mathrm{~S}$ & $<0,0001$ & $\mathrm{~S}$ & $<0,0001$ & $\mathrm{~S}$ & 0,0558 & & 0,3035 & & 0,3842 & \\
\hline M. (A.) burgond-M. (A.) cf. ruffoi & 0,8577 & & $<0,0001$ & S & 0,0028 & S & $<0,0001$ & S & 0,2572 & & $>0,9999$ & \\
\hline M. (A.) valerot.-M. (A.) cf. ruffoi & $<0,0001$ & $\mathrm{~S}$ & $<0,0001$ & s & $<0,0001$ & S & $<0,0001$ & s & $>0,9999$ & & 0,4762 & \\
\hline
\end{tabular}

Tabla 3. Valores de p del test de Scheffe (nivel de significación: 5\%) para cada par de especies y para cada índice utilizado ( $\mathrm{S}=$ diferencia estadísticamente significativa).

Se ha realizado la representación de los centroides de cada una de las poblaciones sobre los planos factoriales definidos por los cuatro ejes retenidos para facilitar la comparación (Figura 3).

El primer eje contrapone las poblaciones de $M$. (A.) burgondiae y de $M$. (A.) cf. ruffoi, con $\mathrm{m} 1$ de gran tamaño, a la de $M$. (A.) valerotensis, con $\mathrm{m} 1$ de pequeño tamaño. Las tres poblaciones restantes se sitúan en posiciones intermedias, ya que tienen $\mathrm{m} 1$ de tamaños similares.

El segundo eje, que expresa un 10,9\% de la varianza, agrupa a las poblaciones en dos conjuntos: el primero incluye a $M$. (A.) pliocaenicus, $M$. (A.) cf. ruffoi y $M$. (A.) valerotensis y se sitúa en la región negativa definida por este eje, y el segundo incluye a $M$. (A.) hintoni nutiensis, $M$. (A.) burgondiae y a la población de la Sima del Elefante y se sitúa en la parte positiva. Este eje está relacionado con la morfología de la región lingual del lóbulo anterior (alta correlación positiva con las variables 9,11 y 13) y con la comunicación entre éste y el rombo pitimiano (correlación alta y de signo opuesto con las variables 17 y 19$)$.

El tercer eje, que expresa un $8,3 \%$ de la varianza, opone Allophaiomys de la Sima del Elefante a las demás poblaciones consideradas. Este eje está relacionado con las variables 8 y 16 , asociadas al desarrollo del esmalte en la parte labial del lóbulo anterior, y con la variable 25 , que expresa la confluencia entre los triángulos T4 y T5.

Se han calculado los ANOVA para la variable 6 (longitud medida sobre el eje de referencia) y los índices establecidos con anterioridad. Se comprueba que en todos los casos existen diferencias significativas entre las poblaciones consideradas (Tabla2). Los test de Scheffe asociados a cada ANOVA permiten establecer las diferencias entre la forma de la Sima del Elefante y las demás especies (Tabla 3).

A partir de estos resultados se aprecia que la población de la Sima del Elefante difiere de las demás especies consideradas en la mayor confluencia entre los 


\begin{tabular}{cccccc}
\hline & $\mathbf{n}$ & med & min & máx & DE \\
\hline $\mathrm{L}$ & 301 & 2,64 & 2,27 & 3,02 & 0,12 \\
$\mathrm{~W}$ & 351 & 0,90 & 0,80 & 1,01 & 0,04 \\
$\mathrm{~A} / \mathrm{L}$ & 301 & 48,74 & 43,55 & 54,54 & 1,43 \\
$\mathrm{~B} / \mathrm{W}$ & 351 & 21,92 & 4,92 & 37,29 & 7,30 \\
$\mathrm{C} / \mathrm{W}$ & 351 & 25,80 & 13,33 & 36,21 & 3,59 \\
\hline
\end{tabular}

Tabla 4. Parámetros de Van der Meulen (1973) para Microtus (Allophaiomys) lavocati nov. sp.

triángulos T4 y T5 (expresada por el índice ICT4T5). Difiere de todas ellas salvo de $M$. (A.) cf. ruffoi en la inclinación del triángulo T4 (expresada por el índice RP), que muestra una orientación más posterior en estas dos especies. Además, difiere de $M$. (A.) pliocaenicus, $M$. (A.) burgondiae, $M$. (A.) cf. ruffoi y, en menor medida, de $M$. (A.) hintoni nutiensis, en el mayor desarrollo de la parte anterior del molar (expresada por el índice LRPA); difiere de $M$. (A.) hintoni nutiensis, $M$. (A.) burgondiae y $M$. (A.) cf. ruffoi en la mayor confluencia entre el rombo pitiminano y el lóbulo anterior (expresada por el índice ICAC2RP); y difiere de $M$. (A.) hintoni nutiensis y, en menor medida, de $M$. (A.) valerotensis y de $M$. (A.) cf. ruffoi, en la mayor simetría transversal de los $\mathrm{m} 1$ (expresada por el índice IAT2T3).

Como resultado de esta comparación, se demuestra que la población de la Sima del Elefante difiere de las demás especies de Allophaiomys consideradas y puede considerarse como una especie nueva, para la que proponemos el nombre de Microtus (Allophaiomys) lavocati nov. sp.

\section{Microtus (Allophaiomys) lavocati nov. sp. Láms. I y II}

Derivatio nominis: dedicada al Prof. René Lavocat, por su extensa y fecunda trayectoria en paleontología de vertebrados.

Holotipo: primer molar inferior derecho (Lám. I, fig. 6) siglado como ATA'96ET10Na 96, depositado en las colecciones del Dpto. de Paleontología de la Universidad de Zaragoza.
Localidad-tipo: Sima del Elefante, unidad roja inferior. El holotipo, así como el resto del material descrito, provienen del nivel ET10Na, excavado en 1996, y que se sitúa en la parte más alta del sondeo realizado con excavadora en ese año, inmediatamente por debajo del nivel del piso de la trinchera, y en el lado norte del relleno cárstico.

Edad: Pleistoceno Inferior.

\section{Material y medidas}

Se dispone de varios miles de $\mathrm{m} 1$ de esta especie provenientes de la unidad roja inferior de la Sima del Elefante. Las medidas de una muestra representativa tomadas según el modelo de Van der Meulen (1973) se expresan en la Tabla 4. Este material se encuentra depositado provisionalmente en las colecciones del Dpto. de Paleontología de la Universidad de Zaragoza.

\section{Diagnosis}

Allophaiomys de talla media, con la parte anterior del $\mathrm{m} 1$ de desarrollo considerable, triángulos T4 y T5 ampliamente confluyentes y triángulo T4 inclinado. Esmalte diferenciado de tipo Microtus.

Medium sized Allophaiomys, with a well-developed anterior part of the $\mathrm{ml}$, wide confluence between triangles T4 and T5, and inclined T4 triangle. Microtuslike enamel differentiation.

\section{Descripción}

Los primeros molares inferiores de $M$. (A.) lavocati nov. sp. constan de un lóbulo posterior, tres triángulos alternos no confluyentes (T1-T3) y un complejo anterior constituido por dos triángulos (T4 y T5) ampliamente confluyentes entre sí y con el lóbulo anterior, de forma redondeada. En éste suele estar presente un $\mathrm{T} 7$ abierto definido por un 5AELi poco desarrollado. El esmalte tiene un recorrido reducido en la región labial del lóbulo anterior y por ello el triángulo T6 sólo está presente en algunos ejemplares. Los triángulos salientes tienen sus extremos redondeados. Los molares son poco asimétricos, con los triángulos labiales algo menos desarrollados que los linguales.

Los terceros molares superiores están constituidos por un lóbulo anterior, dos triángulos no confluyentes (T2 y T3, tipo arrombomorfo de Chaline, 1974) y un complejo posterior en el que el triángulo T4 presenta un grado de confluencia reducido con el lóbulo posterior. En éste se

Lámina I. Morfología de los m1 de Microtus (Allophaiomys) lavocati nov. sp. Todos son molares derechos, excepto los tres últimos, que están invertidos. Escala gráfica: $1 \mathrm{~mm}$.

1 ATA'96ET10Na 1.

2 ATA'96ETNa 20.

3 ATA'96ET10Na 40.

4 ATA'96ET10Na 76.

5 ATA'96ET10Na 81.

6 ATA'96ET10Na 96, holotipo.

7 ATA'96ET10Na 98.

8 ATA'96ET10Na 132.

9 ATA'96ET10Na 147.
10 ATA'96ET10Na 174.

11 ATA'96ET10Na 193.

12 ATA'96ET10Na 201.

13 ATA'96ET10Na 204.

14 ATA'96ET10Na 205.

15 ATA'96ET10Na 230.

16 ATA'96ET10Na 244.

17 ATA'96ET10Na 248. 


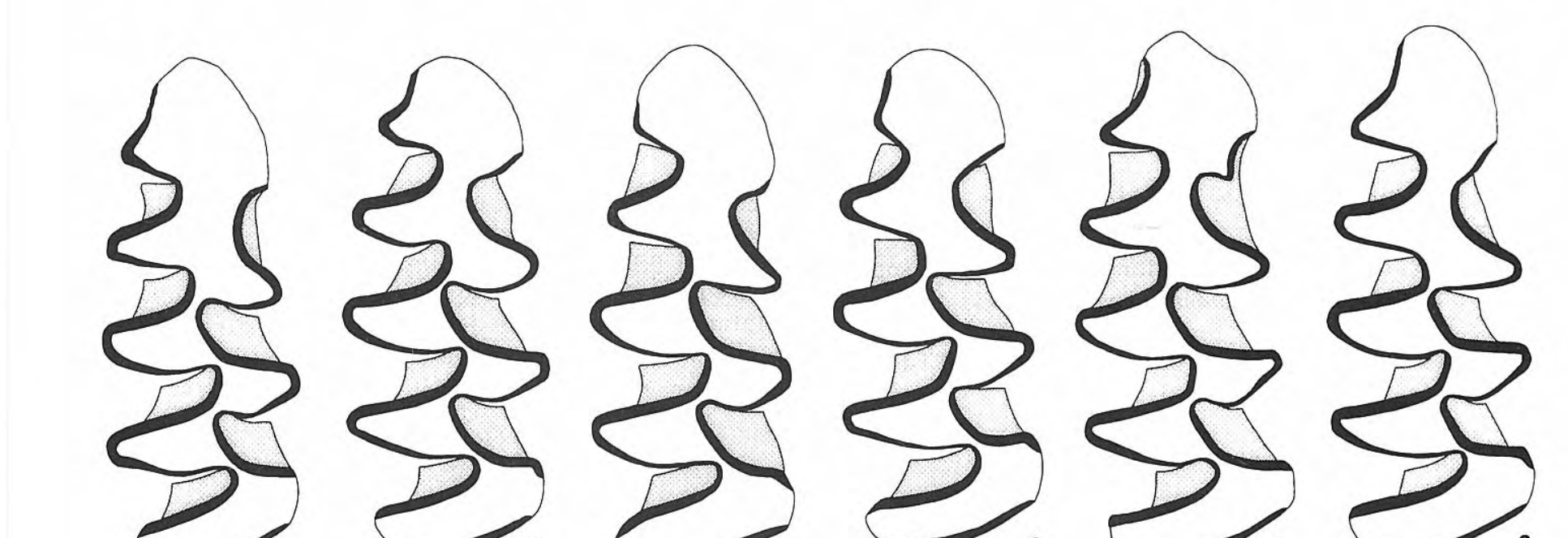

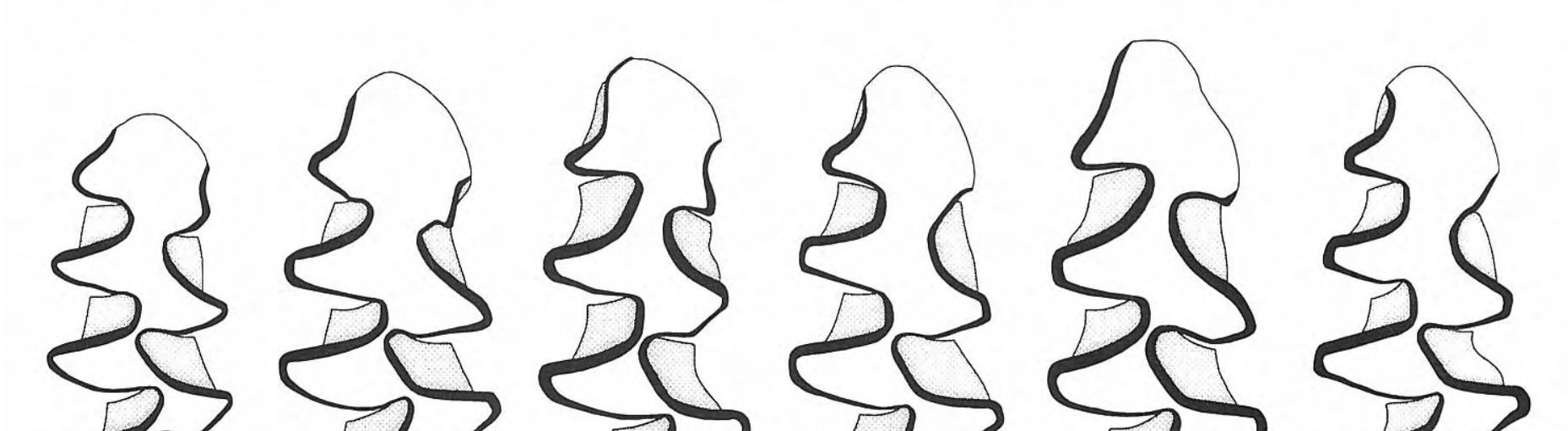

है है दर

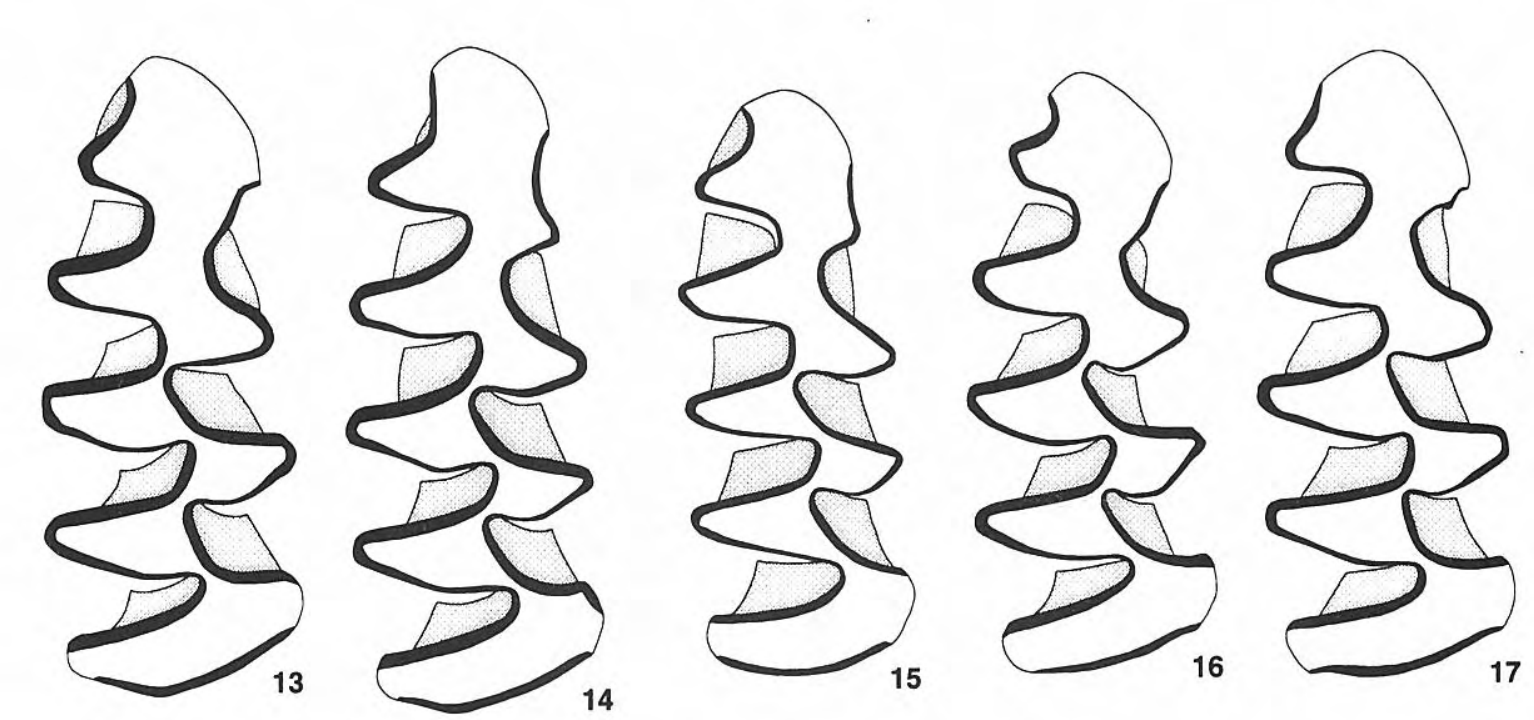


reconoce un T5 generalmente bien desarrollado. El 3AELi es normalmente menos profundo que el 3AELa. Además de las zonas libres de esmalte en el lóbulo anterior y en el extremo más distal del lóbulo posterior, es posible reconocer en algunos ejemplares otras zonas sin esmalte en el extremo de los triángulos linguales T3 y T5.

\section{Comparación con otras especies}

La comparación con $M$. (A.) pliocaenicus de Betfia II, con $M$. (A.) hintoni nutiensis, $M$. (A.) burgondiae y $M$. (A.) valerotensis de Les Valerots, poblaciones-tipo de las especies respectivas, y con $M$. (A.) cf. ruffoi de Cava Pirro ya ha sido realizada en un apartado anterior.

Entre los Allophaiomys descritos en la Península Ibérica, M. (A.) chalinei Alcalde, Agustí y Villalta, 1981 y $M$. (A.) vandermeuleni Agustí, 1991 difieren ampliamente de $M$. (A.) lavocati nov. sp. en la estructura general de los m1. M. (A.) chalinei presenta una talla mayor, un menor desarrollo de la parte anterior del molar, un lóbulo anterior muy simple y aplastado anteroposteriormente y un esmalte de tipo indiferenciado, caracteres que lo diferencian de $M$. (A.) lavocati nov. sp. $M$. (A.) vandermeuleni difiere de $M$. (A.) lavocati nov. sp. en su mayor talla, menor desarrollo de la parte anterior del molar, en la reducción de la confluencia entre los triángulos T4 y T5 y en su esmalte de tipo Mimomys o indiferenciado.

Las poblaciones de Venta Micena (Granada) y Bagur2 (Gerona) fueron inicialmente asignadas a $M$. (A.) pliocaenicus (López et al., 1976; Moyà-Solà et al., 1981). Más tarde, con el mejor conocimiento de la especie italiana $M$. (A.) ruffoi, Agustí (1991) y Masini y Santini (1991) señalan la similitud de la forma de Venta Micena con esta especie. Ruiz Bustos (1988 y 1993) incluye estas dos poblaciones en un nuevo género y una nueva especie, Euphaiomys azarai Ruiz Bustos, 1988, aunque algunos ejemplares de Venta Micena son determinados por este autor como Arvicola deucalion. El holotipo de este nuevo género y especie proviene de Bagur-2. Estos cambios en la atribución taxonómica son el resultado de la consideración por este autor de que el holotipo de $M$. (A.) pliocaenicus pertenece en realidad a un Arvicola primitivo, opinión que no nos parece suficientemente justificada y que a nuestro entender se basa en una división artificial de algunas de las poblaciones asignadas previamente a $M$. (A.) pliocaenicus. Como se ha indicado anteriormente, el arvicólido de Venta Micena se encuentra muy próximo a $M$. (A.) ruffoi, mientras que la asociación de Bagur-2, que presenta una amplia variabilidad morfológica, como ya fue puesto de relieve por López et al. (1976), puede estar constituida por varias especies. Recientemente, Laplana (en prensa) ha identificado la presencia de $M$. (A.) chalinei en este yacimiento. Algunos otros ejemplares guardan similitud con $M$. (A.) lavocati nov. sp., aunque siempre presentan características más primitivas, como una región anterior del m1 menos desarrollada. Una revisión de este material, iniciada por uno de los autores (C.L.), permitirá comprobar si existe alguna relación entre $M$. (A.) lavocati nov. sp. y los ejemplares morfológicamente próximos de Bagur-2.

En Loma-Quemada 1, Agustí et al. (1990) describen un reducido número de ejemplares, asignados a $M$. (A.) burgondiae, con un desarrollo de la parte anterior del $\mathrm{ml}$ similar al de $M$. (A.) lavocati nov. sp., aunque son de talla mayor y tanto las confluencias entre el lóbulo anterior y los triángulos T4-T5 como entre estos triángulos entre sí son más reducidas.

Otras especies de Allophaiomys, como M. (A.) praehintoni Rabeder, 1981, M. (A.) superpliocaenicus Rabeder, 1981 y M. (A.) eoratticeps Paunovic y Rabeder, 1996, definidas en yacimientos del centro de Europa, difieren de $M$. (A.) lavocati nov. sp. en el menor desarrollo de la parte anterior del molar.

\section{Cronología de la asociación de la unidad roja inferior de la Sima del Elefante}

Entre las especies que acompañan a $M$. (A.) lavocati nov. sp. en la unidad roja inferior de la Sima del Elefante, Microtus (Iberomys) aff. huescarensis y Ungaromys nanus pueden aportar algunas precisiones sobre la edad de estos materiales. $M$. (I.) aff. huescarensis es una forma intermedia entre $M$. (A.) hintoni nutiensis de Les Valerots (Francia) y $M$. (I.) huescarensis de los niveles inferiores de Trinchera Dolina (Sierra de Atapuerca, Burgos). El yacimiento de Les Valerots tiene una edad máxima de 1,1 Ma a partir de la datación por ESR de los espeleotemas basales (Chaline, 1984), mientras que los niveles inferiores de Trinchera Dolina tienen una edad mínima de $780 \mathrm{ka}$, al situarse inmediatamente por debajo de la inversión Matuyama-Brunhes (Parés y Pérez González, 1998). Por consiguiente, la edad de la unidad roja inferior de la Sima del Elefante debe situarse entre las estimaciones máxima y mínima de estos dos yacimientos.

Lámina II. Escala gráfica: $1 \mathrm{~mm}$.

1-12 Morfología de los m1 de Microtus (Allophaiomys) lavocati nov. sp.: 1 - ATA'96ET10Na 21; 2 ATA'96ET10Na 48; 3 - ATA'96ET10Na 51; 4 ATA'96ET10Na 56; 5 - ATA'96ET10Na 61; 6 ATA'96ET10Na 66; 7 - ATA'96ET10Na 77; 8 ATA'96ET10Na 85; 9 - ATA'96ET10Na 313; 10 ATA'96ET10Na 150; 11 - ATA'96ET10Na 162; 12 ATA'96ET10Na 168. Todos los molares son derechos, excepto el 9, que está invertido.
13-21 Morfología de los M3 de Microtus (Allophaiomys) lavocati nov. sp.: 13 - ATA'96ET10Na 501; 14 ATA'96ET10Na 502; 15 - ATA'96ET10Na 503; 16 ATA'96ET10Na 504; 17 - ATA'96ET10Na 505; 18 ATA'96ET10Na 506; 19 - ATA'96ET10Na 507; 20 ATA'96ET10Na 508; 21 - ATA'96ET10Na 509. 


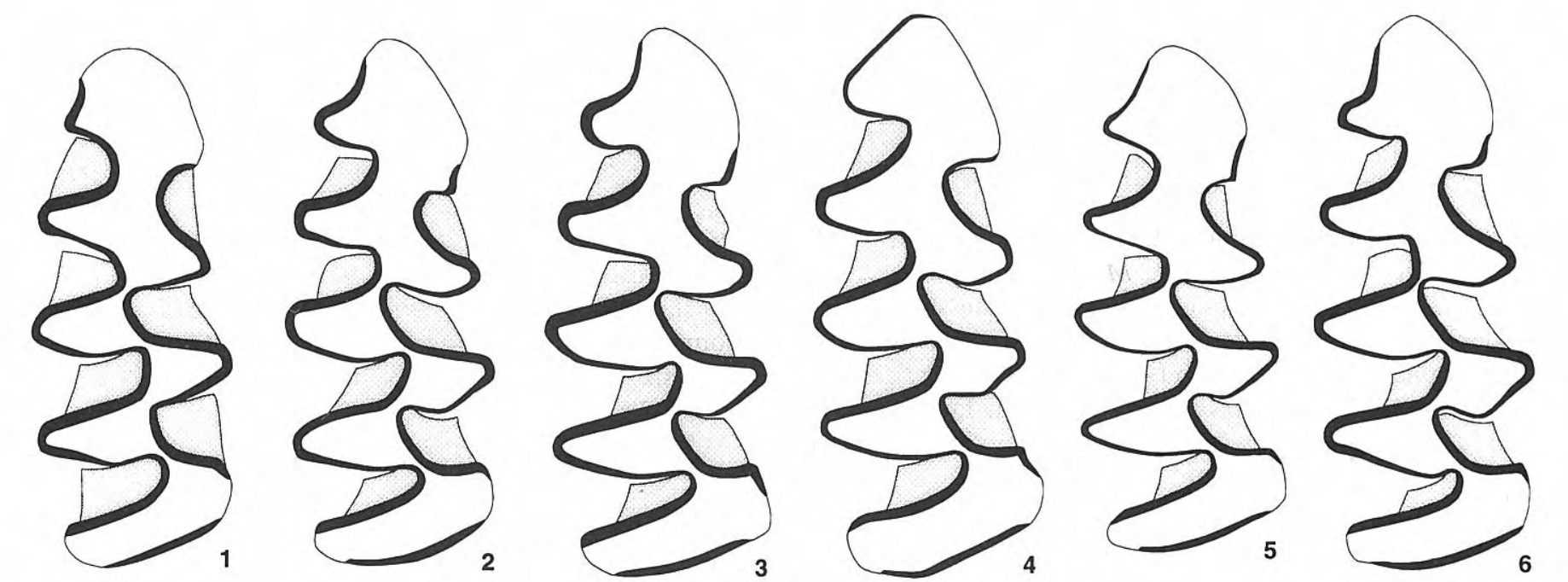

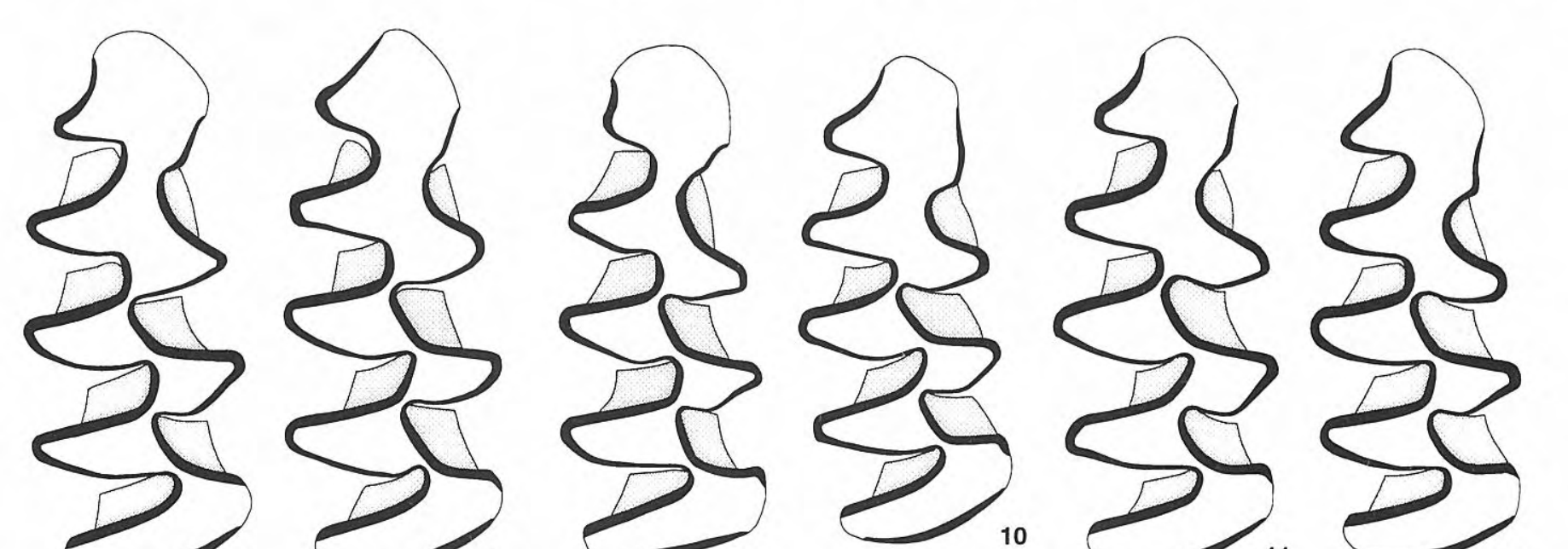

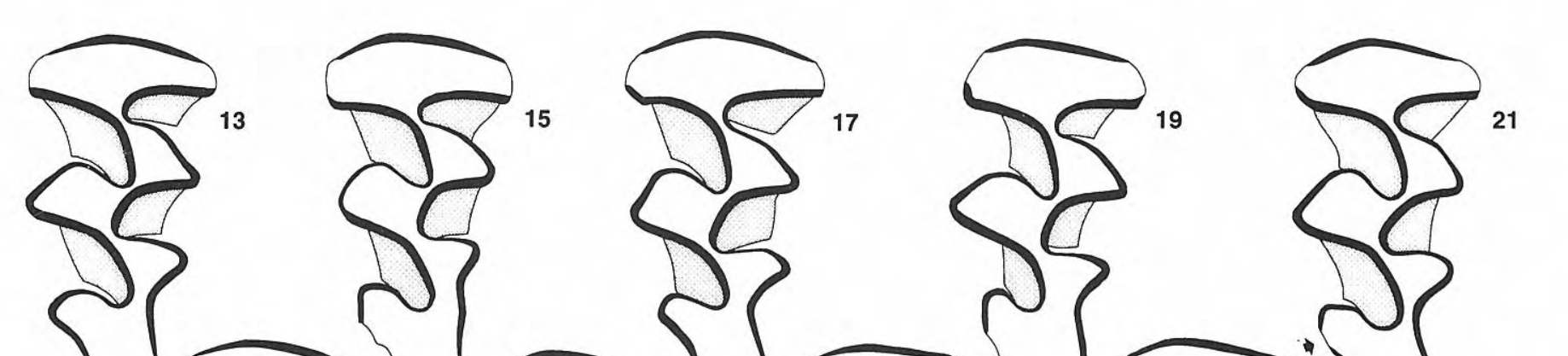

$$
\begin{aligned}
& \text { इड़ }
\end{aligned}
$$


Esta aproximación a la cronología de la Sima del Elefante es confirmada por Ungaromys nanus, un pequeño arvicólido rizodonto característico del Bihariense centroeuropeo. La cita más reciente de esta especie es probablemente la del yacimiento francés de Le Vallonet (Chaline, 1988), con una antigüedad próxima a 1,0 Ma.

Otro criterio de datación relativa alternativo se basa en la tendencia hacia el desarrollo de la parte anterior del $\mathrm{m} 1$ desde las poblaciones de Microtus más primitivas hasta las actuales. Este carácter se encuentra expresado en la mayor parte de las publicaciones recientes a través del índice A/L propuesto por Van der Meulen (1973). Aunque debe ser considerado con precaución, debido a las diferentes tasas de crecimiento en cada una de las líneas evolutivas una vez que éstas se individualizan, puede ser utilizado como indicador del estadio evolutivo de una especie respecto a otras. En este sentido ha sido aplicado recientemente por Maul (1996) y Maul et al. (1998). El valor del índice A/L para $M$. (A.) lavocati nov. sp. es de 48,74, próximo a los de las especies representadas en los yacimientos alemanes de Untermassfeld y Neuleiningen. El dato de Untermassfeld ha sido calibrado con la escala de polaridad magnética, ya que este yacimiento se encuentra en el Subcron Jaramillo (Kahlke en Koenigswald, 1995), y se le asigna una antigüedad próxima a 1,0 Ma. Este resultado concuerda con el obtenido a partir de la asociación de arvicólidos de la unidad roja inferior de la Sima del Elefante.

$M$. (A.) lavocati nov. sp. es por consiguiente una de las especies de Allophaiomys más recientes del Pleistoceno Inferior europeo. Sólo $M$. (A.) chalinei de los niveles inferiores de Trinchera Dolina (Cuenca-Bescós et al., 1995; Laplana y Cuenca-Bescós, 1997) ha sido citado en niveles más modernos que los de la Sima del Elefante.

\section{Relaciones entre $M$. ( A.) lavocati nov. sp. y otras especies de Microtus}

Hasta ahora, las únicas poblaciones conocidas de $M$. (A.) lavocati nov. sp. son las descritas en la Sima del Elefante. Resulta complicado establecer las relaciones de esta especie con otros Allophaiomys debido a la ausencia de otras poblaciones de características similares. Como se ha indicado al hablar de Bagur-2, algunos ejemplares de este yacimiento presentan una morfología similar a la de $M$. (A.) lavocati nov. sp. y podrían representar un estadio más primitivo en la misma dirección evolutiva, pero esta hipótesis no podrá ser confirmada hasta que se realice la revisión de este yacimiento. Lo mismo sucede con los ejemplares determinados como $M$. (A.) pliocaenicus de Saint-Sauveur (Crochet y Michaux, 1978).

Del mismo modo, también se encuentran dificultades para enlazar $M$. (A.) lavocati nov. sp. con otros representantes más modernos del género. La combinación de una amplia confluencia entre los triángulos T4 y T5 y la inclinación del triángulo T4 se encuentra también en Microtus (Terricola) duodecimcostatus (de SélysLongchamps, 1839) y M. (T.) lusitanicus (Gerbe, 1879) desde la parte alta del Pleistoceno Medio. Sin embargo, estas especies se caracterizan por sus M3 de tipo rombomorfo, al contrario que $M$. (A.) lavocati nov. sp.

\section{CONCLUSIONES}

La aplicación del método biométrico propuesto por Brunet-Lecomte (1988) para el estudio de Terricola se ha revelado, con pequeñas adaptaciones, como un instrumento útil en el estudio de otros grupos de arvicólidos, como es el caso de Allophaiomys. Su aplicación a la comparación de las especies $M$. (A.) pliocaenicus, $M$. (A.) cf. ruffoi, $M$. (A.) hintoni nutiensis, $M$. (A.) burgondiae, $M$. (A.) valerotensis y el material de la Sima del Elefante de la Sierra de Atapuerca ha permitido precisar las diferencias entre estos taxones, expresadas en forma de índices en relación con determinados caracteres morfológicos, y confirmar la singularidad de la forma de la Sima del Elefante, para la que se crea la nueva especie $M$. (A.) lavocati nov. sp.

Su asociación en la unidad roja inferior de la Sima del Elefante con $M$. (Iberomys) aff. huescarensis de morfología muy similar, aunque ligeramente más primitiva, a $M$. (I.) huescarensis de los niveles inferiores de Trinchera Dolina, indica para $M$. (A.) lavocati nov. sp. una edad algo más antigua que la de estos niveles, datados como inmediatamente anteriores al límite Pleistoceno Inferior-Medio.

Las relaciones de $M$. (A.) lavocati nov. sp. con otras especies de Microtus anteriores o posteriores permanecen inciertas.

\section{AGRADECIMIENTOS}

Quisiéramos expresar nuestro agradecimiento a los doctores P. Brunet-Lecomte, S. Montuire y al prof. J. Chaline por su amable acogida, hospitalidad y útiles discusiones durante las estancias llevadas a cabo por uno de nosotros (C. L.) en el Laboratorio de Paleontología de la Universidad de Borgoña (Dijon, Francia), realizadas gracias a las Ayudas para Estancias Breves en el Extranjero del programa de Formación de Personal Investigador. Este trabajo se incluye en el proyecto $n^{\circ}$ PB96-1026-C03-02 de la DGICYT, dirigido por José María Bermúdez de Castro (MNCN), y en las actuaciones de la Unidad Asociada al CSIC del Dpto. de Ciencias de la Tierra (Paleontología) de la Universidad de Zaragoza. Agradecemos también el trabajo de los revisores y editores, que ha contribuido a mejorar el texto original.

\section{BIBLIOGRAFÍA}

Agustí, J. 1991. The Allophaiomys complex in Europe. Geobios, 25, 133-144.

Agustí, J., Castillo, C. y Martín-Suárez, E. 1990. Un nuevo yacimiento cárstico del Pleistoceno en la provincia de Granada. Nota preliminar. Actas de Paleontología, 7-24.

Alcalde, G., Agustí, J. y Villalta, J. F. 1981. Un nuevo Allophaiomys (Arvicolidae, Rodentia, Mammalia) en el Pleistoceno Inferior del sur de España. Acta Geologica Hispanica, 16 (4), 203-205.

Brunet-Lecomte, P. 1988. Les campagnols souterrains (Terricola, Arvicolidae, Rodentia) actuels et fossiles 
d'Europe occidentale. Thèse à l'Université de Bourgogne, 1-146.

Carbonell, E., Giralt, S., Márquez, B., Martín, A., Mosquera, A., Ollé, A., Rodríguez, X. P., Sala, R., Vaquero, M., Vergès, J. M. y Zaragoza, J. 1995. El conjunto LitoTécnico de la Sierra de Atapuerca en el marco del Pleistoceno Medio europeo. In: Evolución humana en Europa y los yacimientos de la Sierra de Atapuerca (Eds. J. M. Bermúdez, J. L. Arsuaga y E. Carbonell). Actas de las Jornadas Científicas del Castillo de la Mota (Medina del Campo, Valladolid). Junta de Castilla y León. Vol. 2, 445-556.

Chaline, J. 1972. Les rongeurs du Pléistocène Moyen et Supérieur de France. Cahiers de Paléontologie, 1-410.

Chaline, J. 1974. Esquisse de l'évolution morphologique, biometrique et chromosomique du genre Microtus (Arvicolidae, Rodentia) dans le Pléistocène de l'hémisphère nord. Bulletin de la Société géologique de France, 7, 440-450.

Chaline, J. 1984. La séquence des rongeurs de Bresse, en tant que référence biostratigraphique et paléoclimatique. Géologie de la France, 3, 251-268.

Chaline, J. 1988. Les rongeurs de la Grotte du Vallonet (Alpes-Maritimes), l'environement et l'age du site. L'Anthropologie, 92, 497-499.

Crochet, J.-Y. et Michaux, J. 1978. Une faune de vertébrés du Pléistocène moyen sur le Causse du Larzac: SaintSauveur, près Nant (Aveyron). Paléobiologie continentale, 12, 131-143.

Cuenca-Bescós, G., Canudo, J. I. y Laplana, C. 1995. Los arvicólidos (Rodentia, Mammalia) de los niveles inferiores de Gran Dolina (Pleistoceno Inferior, Atapuerca, Burgos, España). Revista Española de Paleontología, 10, 202-218.

Gerbe, Z. 1879. Diagnose d'un campagnol nouveau de France. Le Naturaliste, 51.

Hír, J. 1998. The Allophaiomys type-material in the Hungarian collection. Paludicola, 2, 28-36.

Koenigswald, W. v. 1995. Mammal traverse. Quaternary vertebrate faunas in Central Europe. In: Quaternary field trips in Central Europe (Ed. W. Schirmer), vol. 2 (13), 707-746.

Kormos, T. 1933. Neue Wühlmäuse aus dem Oberpliozän von Püspökfürdö. Neues Jahrbuch für Mineralogie, Geologie und Paläontologie, B, 69, 323-346.

Laplana, C. (en prensa). Primera cita de Allophaiomys chalinei en el Pleistoceno Inferior de Bagur-2 (Girona, España). Treballs del Museu de Geologia de Barcelona.

Laplana, C. y Cuenca-Bescós, G. 1997. Los arvicólidos (Arvicolidae, Rodentia) del límite Pleistoceno InferiorMedio en el relleno cárstico Trinchera Dolina (Sierra de Atapuerca, Burgos, España). XII Jornadas de Paleontología. Libro de resúmenes y comunicaciones, 192-195.

Laplana, C., Montuire, S., Brunet-Lecomte, P. et Chaline, J. (en prensa). Révision des Allophaiomys (Arvicolinae, Rodentia, Mammalia) des Valerots. Geodiversitas.

López, N., Michaux, J. et Villalta, J. F. 1976. Rongeurs et Lagomorphes de Bagur-2 (Province de Gérone, Espagne), nouveau remplissage de fissure du début du
Pléistocène Moyen. Acta Geologica Hispanica, 11 (2), 46-54.

Martín Suárez, E. and Mein, P. 1991. Revision of the genus Castillomys (Muridae, Rodentia). Scripta Geologica, 96, 47-81.

Masini, F. and Santini, G. 1991. Microtus (Allophaiomys) (Arvicolidae, Rodentia, Mammalia) from Cava Pirro (Apricena, Gargano) and other Italian localities. Bolletino della Societá Paleontologica Italiana, 30, 355-380.

Maul, L. 1996. Biochronological implications of the arvicolids (Mammalia: Rodentia) from the Pliocene and Pleistocene faunas of Neuleiningen (Rheinland-Pfalz, southwest Germany). Acta Zoologica Cracoviensia, 39 (1), 349-356.

Maul, L., Masini, F., Abbazzi, L. and Turner, A. 1998. Geochronometric application of evolutionary trends in the dentition of fossil Arvicolidae. Mededelingen Nederlands Instituut voor Toegepaste Geowetenschappen TNO, 60, 565-572.

Mehely, L. 1914. Fibrinae Hungariae. Die ternären und quartären wurzelzähnigen Wühlmäuse Ungarns. Annales historico naturales Musei Nationalis Hungarici, 12, 155243.

Moyà-Solà, S., Agustí, J., Gibert, J. y Pons-Moyà, J. 1981. El yacimiento cuaternario de Venta Micena (España) y su importancia dentro de las asociaciones faunísticas del Pleistoceno inferior europeo. Paleontologia i evolució, 16, 39-53.

Parés, J. M. y Pérez González, A. 1998. Contexto estratigráfico y cronológico de Gran Dolina (yacimiento de Atapuerca). In: Atapuerca y evolución humana (Ed. E. Aguirre). Fundación Ramón Areces, Madrid, 49-60.

Pasa, A. 1947. I mammiferi di alcune antiche breccie veronesi. Memorie del Museo Civico di Storia Naturale di Verona, 1, 1-111.

Paunovic, M. und Rabeder, G. 1996. Die altpleistozänen Kleinsäugerfaunen Razvodje und Tatinja draga in SüdKroatien. Beiträge zur Paläontologie, 21, 69-84.

Rabeder, G. 1981. Die arvicoliden (Rodentia, Mammalia) aus dem älteren Pleistozän von Niederösterreich. Beiträge zur Paläontologie von Österreich, 8, 1-373.

Ruiz Bustos, A. 1988. Estudio sobre los arvicólidos cuaternarios. Paleomammalia, 2, 1-89.

Ruiz Bustos, A. 1993. New data on Lower Pleistocene arvicolids. The Venta Micena, Betfia-IX and Villany-5 sites. Comunicaciones IX Jornadas de Paleontología, Málaga, 60-64.

Sélys-Longchamps, E. de. 1839. Étude de micromammalogie. Revues des musaraignes, des rats et des campagnols, index méthodiques des mammifères d'Europe. De Roret Ed., Paris, 1-165.

Van der Meulen, A. 1973. Middle Pleistocene Smaller Mammals from the Monte Peglia (Orvieto, Italy), with Special Reference to the Phylogeny of Microtus (Arvicolidae, Rodentia). Quaternaria, 17, 1-144.

Manuscrito recibido: 1 de junio, 1999 Manuscrito aceptado: 7 de septiembre, 1999 